\title{
The Prognostic Value of Neutrophil-to-lymphocyte Ratio and Monocyte-to-lymphocyte Ratio in Metastatic Gastric Cancer Treated with Systemic Chemotherapy
}

\author{
Danyang Zhou ${ }^{1,2}$, Ying $\mathrm{Wu}^{2}$, Ying Zhu ${ }^{1}$, Zhenyu Lin ${ }^{1}$, Dandan $\mathrm{Yu}^{1 * \bowtie}$, Tao Zhang ${ }^{1 * \bowtie}$ \\ 1. Cancer Center, Union Hospital, Tongii Medical College, Huazhong University of Science and Technology, Wuhan 430022, China \\ 2. Department of Medical Oncology, Sun Yat-sen University Cancer Center, State Key Laboratory of Oncology in South China, Collaborative Innovation \\ Center for Cancer Medicine, 651 Dongfeng East Rd, Guangzhou, 510060, China \\ *Equal contribution \\ $\square$ Corresponding authors: Dandan Yu (yudandan@hust.edu.cn), Tao Zhang (taozhangxh@hust.edu.cn)
}

(c) The author(s). This is an open access article distributed under the terms of the Creative Commons Attribution License (https://creativecommons.org/licenses/by/4.0/). See http://ivyspring.com/terms for full terms and conditions.

Received: 2019.08.23; Accepted: 2020.03.27; Published: 2020.04.25

\begin{abstract}
Background: The prognostic value of neutrophil-to-lymphocyte ratio (NLR) and monocyte-tolymphocyte ratio (MLR) in metastatic gastric cancer (mGC) treated with systemic chemotherapy is largely unknown, especially second-line chemotherapy. We retrospectively investigated the prognostic value of baseline NLR and MLR in the progression of mGC with systemic chemotherapy.

Methods: Patients with mGC diagnosed by pathology from January 2010 to December 2018 were identified. Baseline NLR and MLR were collected before treatment. The time to progression during or after first-line therapy from diagnosis (PFS1), and during or after second-line chemotherapy (PFS2) were primary endpoint. Overall survival (OS) was calculated from diagnosis to the date of death or final follow-up.

Results: 537 patients with first-line chemotherapy were included in the retrospective study. The cutoff values of NLR and MLR were 2.610 and 0.285 , respectively. Pretreatment NLR and MLR were significantly independent prognostic factors for PFSI (hazard ratio $[\mathrm{HR}]=1.597,95 \% \mathrm{Cl} 1.261-2.022$, $P<0.001$ and $H R=1.574,95 \% \mathrm{Cl} 1.239-1.999, P<0.001)$ and $O S(H R=1.448,95 \% \mathrm{Cl} 1.030-2.034, P=0.033$ and $\mathrm{HR}=1.622,95 \% \mathrm{Cl} 1.148-2.291, P=0.006)$. For 172 patients treated with second-line chemotherapy, the cutoff value of MLR was 0.355 and MLR maintained a significant association with PFS2 (HR=1.589, 95\% $\mathrm{Cl}$ 1.073-2.354, $P=0.021$ ) in multivariate analysis.

Conclusions: Elevated NLR and MLR were markedly related to the worse PFS1 and OS in mGC performed with first-line chemotherapy. In patients with second-line therapy, MLR was more closely connected to prognosis and was a significantly independent prognostic factor for PFS2.
\end{abstract}

Key words: neutrophil-to-lymphocyte ratio, monocyte-to-lymphocyte ratio, progression-free survival, metastatic gastric cancer, systemic chemotherapy

\section{Introduction}

Gastric cancer (GC) ranks the third for cancer deaths and fifth for cancer incidence worldwide[1]. The death and incidence rates are highest in Asia Pacific[2, 3]. In China, a large proportion of the patients are diagnosed at an advanced stage due to a lack of specific symptoms[4,5]. Despite advances in diagnoses and treatments, the prognosis for metastatic gastric cancers (mGC) remains dismal[6]. A doublet or triplet chemotherapy regimen based fluoropyrimidine and platinum agents is the standard of care for mGC patients with a negative expression of human epidermal growth factor receptor-2 (HER-2) receptor[7, 8] according to the current international guidelines[9]. Many of patients receiving first-line therapy may relapse, modest survival benefit is shown in patients receiving irinotecan, taxane and 
ramucirumab over the best supportive care or active agents for these population[10-13]. However, there are still some mGC patients with the standard therapies and the significantly different prognoses in clinical practice.

Inflammation is an essential component of the tumor microenvironment, and the changes in inflammatory cells have an important role in tumorigenesis, disease progression and patients' prognosis[14, 15]. Peripheral blood tests represent the mirror of the tumor inflammatory conditions. Neutrophil-tolymphocyte ratio (NLR) and monocyte-to-lymphocyte ratio (MLR), as the peripheral blood parameters, are the systemic inflammatory response indicators that have been widely demonstrated to predict outcomes of several solid malignancies[16-21], including our previous data[22]. An increase in the NLR reflects a state of lymphocytopenia and neutrophilia that leads to a protumor microenvironment[23]. Elevated MLR, in fact, prompts to cancer progression through the inhibition of immune system.

In gastric cancer, these hematological parameters also have significantly prognostic values on patients' survival. Li Chen et al. investigated the prognosis of systemic immune-inflammation index, such as platelet-to-lymphocyte ratio (PLR)[24], SII (SII= neutrophil $(\mathrm{N})^{*}$ platelet $(\mathrm{P}) /$ lymphocyte $\left.(\mathrm{L})\right)[25]$ and MLR[26], in patients with GC treated with neoadjuvant chemotherapy. Hua-Long Zheng et al. sought to characterize the effect of the white blood cell to hemoglobin ratio (WHR) on long-term survival after radical gastrectomy[27]. Another study performed by Xiao-dong Chen evaluated the predictive value of PLR for peritoneal metastasis in patients with GC[28]. However, studies on the prognostic values of the NLR and MLR in mGC treated with systemic chemotherapy, especially second-line therapy are rarely reported. Based on this background, the aim of this study was to investigate the prognostic role of the NLR and MLR for survival in mGC patients with first- and second-line strategy.

\section{Methods}

\section{Patients population}

We retrospectively collected clinical data for mGC patients diagnosed pathologically at our Cancer Center between January 2010 and December 2018. Clinical stage of the disease was determined following the $8^{\text {th }}$ American Joint Committee on Cancer guidelines[29]. Patients meeting any of the following criteria were excluded: (1) patients without complete pretreatment baseline parameters; (2) any malignancies besides GC; (3) hematological diseases; (4) evidences of infection or autoimmune diseases; (5) patients without systemic chemotherapy. For all patients, clinical data (age, gender, histology, HER-2 status and chemotherapy regimens) and hematological examination (complete blood count, tumor biomarkers and biochemical parameters) were collected before treatment. Tumor assessment was performed at baseline, at week 8 after chemotherapy, and every 8-12 weeks thereafter, and clinical response was classified according to response evaluation criteria in solid tumors (RECIST 1.1)[30]. All patients included were followed-up regularly until death or study data cutoff (31 December 2018). This retrospective study was approved by the Ethics Committee of Huazhong University of Science and Technology (HUST) in accordance with the ethical standards prescribed by Helsinki Declaration.

\section{Statistical analysis}

Baseline characteristics and treatment strategies of patients were summarized using descriptive statistics. NLR was calculated as the absolute neutrophil count divided by the absolute number of lymphocyte count (NLR=ANC/ALC) and MLR= AMC/ALC. Analysis of receiver operating characteristic (ROC) curves was performed to identify the cutoff value of variables. Median value severed as the cutoff value if area under the curve (AUC) of ROC was less than 0.50. Progression-free survival 1 (PFS1) was determined from diagnosis to disease progression during or after first-line chemotherapy evaluated by imaging, or death (event), or last follow-up (censored). The progression during or after second-line chemotherapy, or death (event), or last follow-up (censored) were defined as progression-free survival 2 (PFS2). Overall survival (OS) was calculated from diagnosis to death (event) or last follow-up (censored). Survival analyses were calculated by the Kaplan-Meier method. Univariate and multivariate analyses were documented using Cox proportional hazards model, and all the significant characteristics on univariate analysis were carried into multivariate analysis. Differences between categorical variables were determined using the Chi-square test. Statistical analysis was performed using SPSS version 21.0. All $P$ values were two-sided, and $P$ values $<0.05$ were considered significant for all statistical analyses.

\section{Result}

\section{Patients treated with first-line chemotherapy}

\section{Clinicopathologic characteristics of patients}

A total of 537 mGC patients who received regularly first-line therapy between January 2010 and December 2018 had available clinicopathological data 
and met the inclusion criteria. Clinical and pathological characteristics of patients were presented in Table 1. The median age at time of diagnosis was 55.0 years (range 25-83 years) and there were 216 $(40.2 \%)$ women and 321 (59.8\%) men. Mean NLR and MLR were 3.060 and 0.320 , respectively. The main first-line chemotherapy schedule was the combination of platinum and fluorouracil $(80.1 \%)$. The median PFS1 and OS were 6.80 and 13.42 months, respectively. The last follow-up time was 31 December 2018.

\section{Prognostic role of baseline NLR and MLR for PFS1 and OS}

NLR and MLR were calculated based on the recommended cutoff values of 2.610 and 0.285 from ROC curves, respectively. On univariate analysis the following variables were found to have prognostic value for PFS1: NLR (hazard ratio [HR] $=1.897,95 \% \mathrm{CI}$ $1.529-2.353, P<0.001)$, MLR (HR=1.795, 95\% CI 1.4432.233, $P<0.001)$, CA125 (HR=1.691, 95\% CI 1.354-2.113, $P<0.001)$, CA199 (HR=1.401, 95\% CI 1.129-1.737, $P=$ $0.002)$, alkaline phosphatase (ALP) (HR=1.268, 95\% CI $1.031-1.559, \quad P=0.025)$. On multivariate analysis showed that elevated NLR (HR=1.597, 95\% CI 1.2612.022, $P<0.001)$, MLR (HR=1.574, 95\% CI 1.239-1.999, $P<0.001)$, CA125 (HR=1.559, 95\% CI 1.238-1.964, $P<0.001)$ and CA199 (HR=1.256, 95\% CI 1.003-1.572, $P=0.047)$ were the variables independently associated with shortened survival (Table 2). Similar results were revealed in the relationships of these factors with OS.
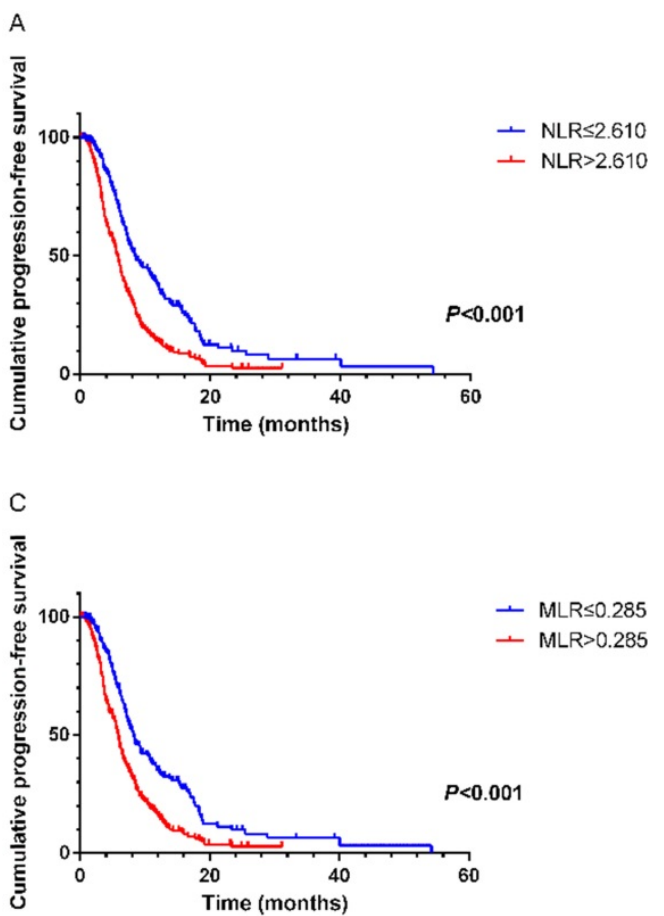

Multivariate analysis demonstrated that pretreatment NLR (HR=1.448, 95\% CI 1.030-2.034, $P=0.033)$, MLR (HR=1.622, 95\% CI 1.148-2.291, $P=0.006)$ and CA125 (HR=1.675, 95\% CI 1.267-2.215, $P<0.001)$ were significantly correlated with OS (Table 3). Kaplan-Meier survival curves also suggested that NLR and MLR had close links with PFS1 and OS (Figure 1).

Table 1. General characteristics of $\mathrm{mGC}$ patients undergoing first-line chemotherapy $(n=537)$

\begin{tabular}{ll}
\hline Variable & Value \\
\hline Age (Year) & $55.0(25-83)$ \\
Gender (Female/male) & $216 / 321$ \\
Histology (Well/moderately/poorly & $6 / 38 / 303 / 190$ \\
differentiated/unknown) & \\
HER-2 (Positive) (available in 210 pts) & $22(10.5 \%)$ \\
First-line chemotherapy & \\
Platinum + fluorouracil & $430(80.1 \%)$ \\
Others & $107(19.9 \%)$ \\
NLR & $3.060(0.12-58.23)$ \\
PLR & $196.0(21-886)$ \\
MLR & $0.320(0.05-1.91)$ \\
CA125 (U/ml) & $30.75(3.0-4853.6)$ \\
CA199 (U/ml) & $17.70(1.0-12000.0)$ \\
CEA (ug/L) & $3.45(0.5-11112.9)$ \\
ALB (g/L) & $37.70(15.9-54.4)$ \\
GLB (g/L) & $26.70(15.1-51.7)$ \\
ALP (U/L) & $73.0(27-3229)$ \\
Progress-free survival 1 & $6.80(0.1-54.2)$ \\
Overall survival & $13.42(0.1-73.2)$ \\
\hline
\end{tabular}

Abbreviations: NLR: neutrophil-to-lymphocyte ratio; PLR: platelet-to-lymphocyte ratio; MLR: monocyte-to-lymphocyte ratio; ALB: albumin; GLB: globulin; ALP: alkaline phosphatase; Progress-free survival 1: the time to progression after first-line from diagnosis.

B

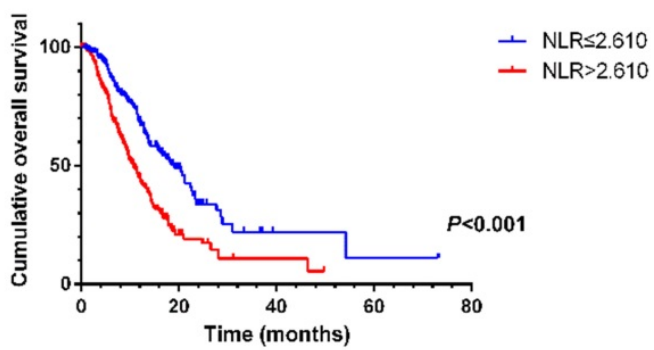

D

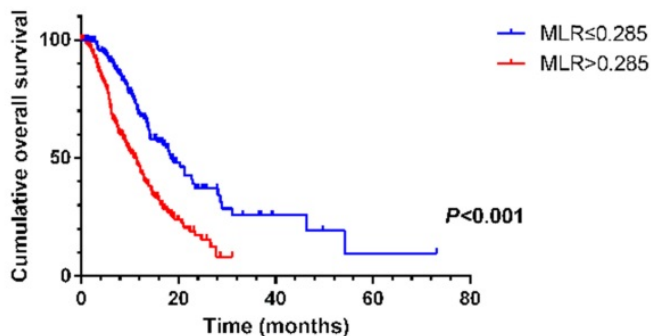

Figure 1. A. Kaplan-Meier curve for PFSI of mGC patients with first-line chemotherapy stratified by NLR. B. Kaplan-Meier curve for OS of mGC patients with first-line chemotherapy stratified by NLR. C. Kaplan-Meier curve for PFSI of mGC patients with first-line chemotherapy stratified by MLR. D. Kaplan-Meier curve for OS of mGC patients with first-line chemotherapy stratified by MLR. 
Table 2. Cox proportional hazard regression analysis of progression-free survival in mGC patients undergoing first-line chemotherapy $(n=573)$

\begin{tabular}{|c|c|c|c|c|}
\hline \multirow[t]{2}{*}{ Faction } & \multicolumn{2}{|l|}{ Univariable } & \multicolumn{2}{|l|}{ Multivariable } \\
\hline & HR (95\% CI) & $P$ & HR $(95 \% \mathrm{CI})$ & $P$ \\
\hline Age $(>55.0$ vs $\leq 55.0)$ & $0.976(0.795-1.198)$ & 0.818 & & \\
\hline Gender (M vs F) & $0.997(0.809-1.228)$ & 0.975 & & \\
\hline Histology (Well vs moderately vs poorly differentiated) & $1.192(0.876-1.624)$ & 0.264 & & \\
\hline HER-2 (Positive vs negative) & $1.330(0.776-2.279)$ & 0.300 & & \\
\hline $\operatorname{NLR}(>2.610$ vs $\leq 2.610)$ & $1.897(1.529-2.353)$ & $<0.001$ & $1.597(1.261-2.022)$ & $<0.001$ \\
\hline PLR $(>284.0$ vs $\leq 284.0)$ & $1.227(0.960-1.569)$ & 0.103 & & \\
\hline $\operatorname{MLR}(>0.285$ vs $\leq 0.285)$ & $1.795(1.443-2.233)$ & $<0.001$ & $1.574(1.239-1.999)$ & $<0.001$ \\
\hline CA125 (> 25.90 vs $\leq 25.90 \mathrm{U} / \mathrm{ml})$ & $1.691(1.354-2.113)$ & $<0.001$ & 1.559 (1.238-1.964) & $<0.001$ \\
\hline CA199 (> 17.70 vs $\leq 17.70 \mathrm{U} / \mathrm{ml})$ & 1.401 (1.129-1.737) & 0.002 & $1.256(1.003-1.572)$ & 0.047 \\
\hline CEA $(>4.55$ vs $\leq 4.55 \mathrm{ug} / \mathrm{L})$ & $1.205(0.975-1.489)$ & 0.085 & & \\
\hline $\operatorname{ALB}(>39.25$ vs $\leq 39.25 \mathrm{~g} / \mathrm{L})$ & $0.928(0.742-1.161)$ & 0.515 & & \\
\hline GLB (> 26.70 vs $\leq 26.70 \mathrm{~g} / \mathrm{L})$ & $1.177(0.953-1.452)$ & 0.130 & & \\
\hline $\operatorname{ALP}(>78.5$ vs $\leq 78.5 \mathrm{U} / \mathrm{L})$ & $1.268(1.031-1.559)$ & 0.025 & $1.144(0.911-1.437)$ & 0.246 \\
\hline
\end{tabular}

Abbreviations: NLR: neutrophil-to-lymphocyte ratio; PLR: platelet-to-lymphocyte ratio; MLR: monocyte-to-lymphocyte ratio; ALB: albumin; GLB: globulin; ALP: alkaline phosphatase. $P$ less than 0.05 is statistically significant.

Table 3. Cox proportional hazard regression analysis of overall survival in $m G C$ patients undergoing first-line chemotherapy $(n=573)$

\begin{tabular}{|c|c|c|c|c|}
\hline \multirow[t]{2}{*}{ Faction } & \multicolumn{2}{|l|}{ Univariable } & \multicolumn{2}{|l|}{ Multivariable } \\
\hline & HR (95\% CI) & $P$ & HR (95\% CI) & $P$ \\
\hline Age $(>55.0$ vs $\leq 55.0)$ & $1.157(0.899-1.488)$ & 0.257 & & \\
\hline Gender (M vs F) & $0.909(0.706-1.171)$ & 0.461 & & \\
\hline Histology (Well vs moderately vs poorly differentiated) & $0.922(0.609-1.396)$ & 0.701 & & \\
\hline HER-2 (Positive vs negative) & $1.240(0.659-2.331)$ & 0.505 & & \\
\hline NLR $(>2.610$ vs $\leq 2.610)$ & $2.034(1.555-2.661)$ & $<0.001$ & $1.448(1.030-2.034)$ & 0.033 \\
\hline PLR (> 284.0 vs $\leq 284.0)$ & $1.452(1.080-1.953)$ & 0.014 & $1.016(0.733-1.406)$ & 0.926 \\
\hline $\operatorname{MLR}(>0.285$ vs $\leq 0.285)$ & $2.218(1.678-2.930)$ & $<0.001$ & $1.622(1.148-2.291)$ & 0.006 \\
\hline CA125 (> 25.90 vs $\leq 25.90 \mathrm{U} / \mathrm{ml})$ & $1.909(1.453-2.508)$ & $<0.001$ & $1.675(1.267-2.215)$ & $<0.001$ \\
\hline CA199 (> 17.70 vs $\leq 17.70 \mathrm{U} / \mathrm{ml})$ & $1.275(0.982-1.655)$ & 0.068 & & \\
\hline CEA $(>4.55 \mathrm{vs} \leq 4.55 \mathrm{ug} / \mathrm{L})$ & $1.545(1.195-1.998)$ & 0.001 & & \\
\hline $\operatorname{ALB}(>39.25 \mathrm{vs} \leq 39.25 \mathrm{~g} / \mathrm{L})$ & $0.759(0.574-1.003)$ & 0.052 & & \\
\hline GLB (> 26.70 vs $\leq 26.70 \mathrm{~g} / \mathrm{L})$ & $0.930(0.715-1.210)$ & 0.591 & & \\
\hline $\mathrm{ALP}(>78.5 \mathrm{vs} \leq 78.5 \mathrm{U} / \mathrm{L})$ & $1.156(0.896-1.491)$ & 0.265 & & \\
\hline
\end{tabular}

Abbreviations: NLR: neutrophil-to-lymphocyte ratio; PLR: platelet-to-lymphocyte ratio; MLR: monocyte-to-lymphocyte ratio; ALB: albumin; GLB: globulin; ALP: alkaline phosphatase. $P$ less than 0.05 is statistically significant.

\section{Associations of NLR and MLR with other clinicopathologic parameters}

Baseline NLR and MLR as promising prognostic factors in mGC patients conducted with first-line chemotherapy, we subsequently performed a comparison of the clinicopathologic characteristics according to NLR and MLR, respectively. The following variables related to elevated NLR: high CA125 $(P<0.001)$, CEA $(P<0.001)$, globulin $(\mathrm{GLB})$ $(P=0.031)$ and ALP $(P=0.001)$. CA125 $(P<0.001)$, albumin (ALB) $(P=0.011)$ and ALP $(P=0.002)$ had significant relations with MLR.

\section{Patients treated with second-line chemotherapy}

Clinicopathologic characteristics of patients

Among 537 mGC patients with first-line chemotherapy, 172 (32.0\%) patients conducted the second-line chemotherapy after disease progression which is consistent with previous statistics[31]. Could NLR and/or MLR be beneficial in predicting the development of tumor for these patients? Baseline data of 172 patients were shown in Table 4 . The main first-line schedule was also a doublet chemotherapy regimen-based fluorouracil and platinum agents $(87.2 \%)$. The second-line regimen was following: chemotherapy based taxane (45.3\%) and chemotherapy-based platinum (16.9\%), chemotherapy-based irinotecan $(16.3 \%)$ and others $(21.5 \%)$. The mean values of NLR and MLR were 3.030 and 0.310, respectively. The median month of PFS2 was 3.70 months.

\section{Prognostic role of baseline NLR and MLR for PFS2}

According to ROC curves, the cutoff values of NLR and MLR were 3.110 and 0.355 , respectively. Significant characteristics on univariate analysis (NLR (HR=1.588, 95\% CI 1.102-2.287, $P=0.013)$, MLR $(\mathrm{HR}=1.703,95 \%$ CI 1.178-2.463, $P=0.005)$ and CA125 ( $\mathrm{HR}=1.602,95 \%$ CI 1.075-2.389, $P=0.021)$ ) were carried into multivariate analysis. MLR (HR=1.589, 95\% CI 1.073-2.354, $P=0.021)$ and CA125 (HR=1.564, 95\% CI 1.043-2.346, $P=0.031)$ ) were significantly associated 
with PFS2 by multivariate analysis (Table 5). Using Kaplan-Meier analysis, elevated NLR and MLR predicted a shorter disease progression, although NLR was not an independent prognostic factor (Figure 2).

\section{Associations of MLR with other clinicopathologic parameters}

In analyzing the correlation between MLR and clinicopathologic factors, CA125 $(P=0.098)$ seemed to be different among the two groups stratified by MLR, but not statistically significance.

\section{Discussion}

In this study, we aimed to identify clinically useful inflammation-based prognostic indicators for individualized treatments in mGC patients undergoing systemic chemotherapy and most likely to help these patients to benefit from current chemotherapeutic strategies. On the base of analysis on patients with first-line chemotherapy, we further explored the prognostic value of systemic inflammatory response indicators, namely NLR and MLR in patients accepted with second-line chemotherapy. Present study demonstrated that baseline NLR and MLR were independently predictive factors for $\mathrm{mGC}$ treated with first-line chemotherapy. For mGC patients performed with second-line therapy, MLR possessed better prognostic value for disease progression and therapeutic efficiency than NLR.

Table 4. General characteristics of $m G C$ patients undergoing second-line chemotherapy $(n=172)$

\begin{tabular}{ll}
\hline Variable & Value \\
\hline Age (Year) & $53.0(27-76)$ \\
Gender (Female/male) & $72 / 100$ \\
Histology (Well/moderately/poorly & $2 / 16 / 98 / 56$ \\
differentiated/not known) & \\
HER-2 (Positive) (available in 73 pts) & $8(11.0 \%)$ \\
First-line chemotherapy & \\
Platinum + fluorouracil & $150(87.2 \%)$ \\
Others & $22(12.8 \%)$ \\
Second-line chemotherapy & \\
Chemotherapy-based taxane & $78(45.3 \%)$ \\
Chemotherapy-based platinum & $29(16.9 \%)$ \\
Chemotherapy-based irinotecan & $28(16.3 \%)$ \\
Others & $37(21.5 \%)$ \\
NLR & $3.030(0.92-12.91)$ \\
PLR & $196.0(38-620)$ \\
MLR & $0.310(0.08-1.35)$ \\
CA125 (U/ml) & $34.60(6.5-1061.7)$ \\
CA199 (U/ml) & $18.10(1.0-12000.0)$ \\
CEA (ug/L) & $2.80(0.5-11112.9)$ \\
ALB (g/L) & $37.90(20.0-54.4)$ \\
GLB (g/L) & $26.35(16.5-48.7)$ \\
ALP (U/L) & $74.5(37-468)$ \\
Progress-free survival 2 & $3.70(0.1-29.9)$ \\
\hline Abbreviations: NLR: neutrophil-to-lymphocyte ratio; PLR: platelet-to-lymphocyte \\
ratio; MLR: monocyte-to-lymphocyte ratio; ALB: albumin; GLB: globulin; ALP: \\
alkaline phosphatase; Progress-free survival 2: the time to progression after \\
second-line chemotherapy. \\
\end{tabular}

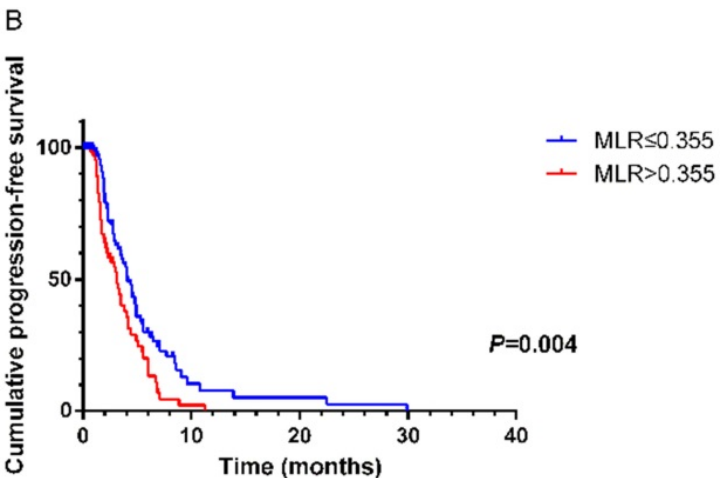

Figure 2. Kaplan-Meier curve for PFS2 of mGC patients with second-line chemotherapy stratified by NLR (A) and MLR (B).

Table 5. Cox proportional hazard regression analysis of progression-free survival in $\mathrm{mGC}$ patients undergoing second-line chemotherapy $(n=172)$

\begin{tabular}{|c|c|c|c|c|}
\hline \multirow[t]{2}{*}{ Faction } & \multicolumn{2}{|l|}{ Univariable } & \multicolumn{2}{|l|}{ Multivariable } \\
\hline & HR (95\% CI) & $P$ & HR $(95 \%$ CI $)$ & $P$ \\
\hline Age $(>53.0$ vs $\leq 53.0)$ & $0.950(0.662-1.364)$ & 0.781 & & \\
\hline Gender (M vs F) & $1.262(0.874-1.820)$ & 0.214 & & \\
\hline Histology (Well vs moderately vs poorly differentiated) & $0.896(0.555-1.449)$ & 0.655 & & \\
\hline HER-2 (Positive vs negative) & $0.953(0.375-2.420)$ & 0.919 & & \\
\hline NLR $(>3.110$ vs $\leq 3.110)$ & $1.588(1.102-2.287)$ & 0.013 & $1.435(0.975-2.112)$ & 0.067 \\
\hline PLR (> 129.0 vs $\leq 129.0)$ & $0.973(0.630-1.503)$ & 0.903 & & \\
\hline MLR $(>0.355$ vs $\leq 0.355)$ & $1.703(1.178-2.463)$ & 0.005 & $1.589(1.073-2.354)$ & 0.021 \\
\hline CA125 (> $34.60 \mathrm{vs} \leq 34.60 \mathrm{U} / \mathrm{ml})$ & $1.602(1.075-2.389)$ & 0.021 & $1.564(1.043-2.346)$ & 0.031 \\
\hline CA199 (> 18.10 vs $\leq 18.10 \mathrm{U} / \mathrm{ml})$ & $1.171(0.799-1.717)$ & 0.418 & & \\
\hline $\mathrm{CEA}(>2.80 \mathrm{vs} \leq 2.80 \mathrm{ug} / \mathrm{L})$ & $1.386(0.950-2.023)$ & 0.091 & & \\
\hline
\end{tabular}




\begin{tabular}{|c|c|c|c|c|}
\hline \multirow[t]{2}{*}{ Faction } & \multicolumn{2}{|l|}{ Univariable } & \multicolumn{2}{|l|}{ Multivariable } \\
\hline & HR $(95 \%$ CI) & $P$ & HR $(95 \%$ CI) & $P$ \\
\hline $\operatorname{ALB}(>38.00$ vs $\leq 38.00 \mathrm{~g} / \mathrm{L})$ & $0.707(0.478-1.045)$ & 0.082 & & \\
\hline GLB $(>26.35$ vs $\leq 26.35 \mathrm{~g} / \mathrm{L})$ & $1.078(0.742-1.566)$ & 0.692 & & \\
\hline $\operatorname{ALP}(>74.5$ vs $\leq 74.5 \mathrm{U} / \mathrm{L})$ & $1.259(0.875-1.814)$ & 0.215 & & \\
\hline
\end{tabular}

Abbreviations: NLR: neutrophil-to-lymphocyte ratio; PLR: platelet-to-lymphocyte ratio; MLR: monocyte-to-lymphocyte ratio; ALB: albumin; GLB: globulin; ALP: alkaline phosphatase. $P$ less than 0.05 is statistically significant.

It is widely recognized that inflammation promotes the development of tumor, such as Helicobacter pylori infection and the risk of gastric cancer[32]. On the other hand, tumor can also trigger regional inflammatory responses and release proinflammatory cytokines leading to the formation of an inflammatory microenvironment[33-35]. Elevated NLR reflects a state of neutrophilia and/or lymphocytopenia and high MLR presents increased monocyte and/or decreased lymphocyte. Neutrophils and monocytes are linked to induce angiogenesis, inflammation and pro-tumor immune response by secreting a variety of cytokines and cytotoxic mediators[36-38]. Tumor-infiltrating lymphocytes, as a type of lymphocytes, are the most important mediators of an anti-tumor immune response[39, 40].

In our study, elevated NLR and MLR predict the worse PFS1 and OS which is constant with the previous researches[20, 41]. For mGC patients, the survival analysis of systemic chemotherapy is superior to best supportive care in several randomized studies[42, 43]. Therefore, subgroup analysis of prognostic factors for mGC treated with systemic chemotherapy are more clinically valuable than that of advanced patients regardless of treatment or not. For patients with first-line chemotherapy, NLR and MLR serves as prognostic factors to evaluate the treatment effectiveness and the survival. Van Soest RJ et al.[44], Bruix J et al.[45] and Choi YH et al.[46] demonstrated the similar results in solid cancer with systemic chemotherapy. NLR and MLR are also related to CA125[47] and ALP[48] that are widely demonstrated to associate with the prognosis of solid cancers. Tumor markers are related to tumor burden[49] and elevated serum ALP are known to be associated with poor survival in locally advanced and metastatic cancers[50].

Many of patients receiving first-line therapy relapse over time and second-line treatment remains the only available option for disease progression[51]. For these patients, the prognostic factors for disease progression and therapeutic efficiency are controversial. In present study, MLR is a significantly independent factor in mGC with second-line chemotherapy. Unfortunately, NLR has no significant association with disease progression in these people. However, little is known about the mechanism of NLR and MLR and its association with prognosis in metastatic cancer with chemotherapy. Neutropenia and its complications remain major toxicities related to myelosuppressive chemotherapy in several solid tumors[52, 53]. With the increasing cycles of chemotherapy, bone marrow toxicities accumulate and immune status alters[54, 55]. As responders in inflammation, neutrophils rapidly migrate between the blood stream and the tissue. Recent discoveries have revealed the ability of these cells to shape the immune response by playing an important role at the interface between innate and adaptive immunity[56]. This may partly explain why NLR lose the prognosis in $\mathrm{mGC}$ with second-line therapy. Reported previously, hepatocyte growth factor receptor (c-Met) [57], immune checkpoints (PD-1 and PD-L1)[58], BRCA mutations[59] and fibroblast growth factor receptors (FGFR) and their ligands[60] are the most studied biomarkers for survival and make breakthroughs. MLR, as a saving-time, inexpensive and valid biomarker, has its advantages in clinical practice. To the best of our knowledge, our current study is the first to show that MLR is a promising factor for evaluating the prognosis in patients with mGC who undergone second-line chemotherapy.

Regrettably, NLR and MLR have no significate link to HER-2 expression both first- and second-line chemotherapy. Other prognostic markers need to be explored for mGC with a positive expression of HER2 receptor who conducted with systemic treatment.

Nevertheless, we should be clear that there are some limitations in addition to the single institution and retrospective study design. The present findings need to be validated using a larger cohort of patients and subgroup analysis between different second-line regimens.

\section{Conclusion}

Although systemic chemotherapy is the first choice for mGC patients, the prognostic factors for survival and efficiency are yet to be explored. Pretreatment NLR and MLR were markedly related to the progression and survival in mGC with first-line chemotherapy, while MLR are more promising in patients with second-line treatment. NLR and MLR, as simple, inexpensive and readily available biomarkers, if proper combination and application, help predict response to chemotherapy and survival in mGC patients with systemic chemotherapy. 


\section{Acknowledgements}

The research was supported by the National Natural Science Foundation of China (No. 81874061 and No. 81872429).

\section{Ethical Standards}

This retrospective study is approved by the Ethical Committees.

\section{Competing Interests}

The authors have declared that no competing interest exists.

\section{References}

1. Bray F, Ferlay J, Soerjomataram I, Siegel RL, Torre LA, Jemal A. Global cancer statistics 2018: GLOBOCAN estimates of incidence and mortality worldwide for 36 cancers in 185 countries. CA: a cancer journal for clinicians. 2018; 68: 394-424.

2. Fitzmaurice C, Dicker D, Pain A, Hamavid H, Moradi-Lakeh M, MacIntyre MF, et al. The Global Burden of Cancer 2013. JAMA oncology. 2015; 1: 505-27.

3. Torre LA, Bray F, Siegel RL, Ferlay J, Lortet-Tieulent J, Jemal A. Global cancer statistics, 2012 CA: a cancer journal for clinicians. 2015; 65: 87-108.

4. Chen L, Zuo Y, Zhu L, Zhang Y, Li S, Ma F, et al. Peripheral venous blood neutrophil-to-lymphocyte ratio predicts survival in patients with advanced gastric cancer treated with neoadjuvant chemotherapy. OncoTargets and therapy. 2017; 10: 2569-80.

5. Digklia A, Wagner AD. Advanced gastric cancer: Current treatment landscape and future perspectives. World journal of gastroenterology. 2016; 22: 2403-14

6. Wang J, Sun Y, Bertagnolli MM. Comparison of gastric cancer survival between Caucasian and Asian patients treated in the United States: results from the Surveillance Epidemiology and End Results (SEER) database. Annals of surgical oncology. 2015; 22: 2965-71.

7. Cunningham D, Starling N, Rao S, Iveson T, Nicolson M, Coxon F, et al. Capecitabine and oxaliplatin for advanced esophagogastric cancer. The New England journal of medicine. 2008; 358: 36-46.

8. Van Cutsem E, Moiseyenko VM, Tjulandin S, Majlis A, Constenla M, Boni C, et al. Phase III study of docetaxel and cisplatin plus fluorouracil compared with cisplatin and fluorouracil as first-line therapy for advanced gastric cancer: a report of the V325 Study Group. Journal of clinical oncology: official journal of the American Society of Clinical Oncology. 2006; 24: 4991-7.

9. Smyth EC, Verheij M, Allum W, Cunningham D, Cervantes A, Arnold D. Gastric cancer: ESMO Clinical Practice Guidelines for diagnosis, treatment and follow-up. Annals of oncology: official journal of the European Society for Medical Oncology. 2016; 27: v38-v49.

10. Thuss-Patience PC, Kretzschmar A, Bichev D, Deist T, Hinke A, Breithaupt K, et al. Survival advantage for irinotecan versus best supportive care as second-line chemotherapy in gastric cancer--a randomised phase III study of the Arbeitsgemeinschaft Internistische Onkologie (AIO). European journal of cancer (Oxford, England: 1990). 2011; 47: 2306-14.

11. Kang JH, Lee SI, Lim DH, Park KW, Oh SY, Kwon HC, et al. Salvage chemotherapy for pretreated gastric cancer: a randomized phase III trial comparing chemotherapy plus best supportive care with best supportive care alone. Journal of clinical oncology: official journal of the American Society of Clinical Oncology. 2012; 30: 1513-8.

12. Ford HE, Marshall A, Bridgewater JA, Janowitz T, Coxon FY, Wadsley J, et al. Docetaxel versus active symptom control for refractory oesophagogastric adenocarcinoma (COUGAR-02): an open-label, phase 3 randomised controlled trial. The Lancet Oncology. 2014; 15: 78-86.

13. Fuchs CS, Tomasek J, Yong CJ, Dumitru F, Passalacqua R, Goswami C, et al. Ramucirumab monotherapy for previously treated advanced gastric or gastro-oesophageal junction adenocarcinoma (REGARD): an international, randomised, multicentre, placebo-controlled, phase 3 trial. Lancet (London, England). 2014; 383: 31-9.

14. Gabrilovich DI, Ostrand-Rosenberg S, Bronte V. Coordinated regulation of myeloid cells by tumours. Nature reviews Immunology. 2012; 12: 253-68.

15. Diakos CI, Charles KA, McMillan DC, Clarke SJ. Cancer-related inflammation and treatment effectiveness. The Lancet Oncology. 2014; 15: e493-503.

16. Feng F, Sun L, Zheng G, Liu S, Liu Z, Xu G, et al. Low lymphocyte-to-white blood cell ratio and high monocyte-to-white blood cell ratio predict poor prognosis in gastric cancer. Oncotarget. 2017; 8: 5281-91.

17. Zheng J, Cai J, Li H, Zeng K, He L, Fu H, et al. Neutrophil to Lymphocyte Ratio and Platelet to Lymphocyte Ratio as Prognostic Predictors for Hepatocellular Carcinoma Patients with Various Treatments: a Meta-Analysis and Systemic Review. Cellular physiology and biochemistry: international journal of experimental cellular physiology, biochemistry, and pharmacology. 2017; 44: $967-81$
18. Ethier JL, Desautels D, Templeton A, Shah PS, Amir E. Prognostic role of neutrophil-to-lymphocyte ratio in breast cancer: a systemic review and meta-analysis. Breast cancer research: BCR. 2017; 19: 2.

19. Li MX, Liu XM, Zhang XF, Zhang JF, Wang WL, Zhu Y, et al. Prognostic role of neutrophil-to-lymphocyte ratio in colorectal cancer: a systemic review and meta-analysis. International journal of cancer. 2014; 134: 2403-13

20. Huang OT, Zhou L, Zeng WJ, Ma OQ, Wang W, Zhong M, et al. Prognostic Significance of Neutrophil-to-Lymphocyte Ratio in Ovarian Cancer: A Systemic Review and Meta-Analysis of Observational Studies. Cellular physiology and biochemistry : international journal of experimental cellular physiology, biochemistry, and pharmacology. 2017; 41: 2411-8.

21. Fu H, Zheng J, Cai J, Zeng K, Yao J, Chen L, et al. Systemic Immune-Inflammation Index (SII) is Useful to Predict Survival Outcomes in Patients After Liver Transplantation for Hepatocellular Carcinoma within Hangzhou Criteria. Cellular physiology and biochemistry: international journal of experimental cellular physiology, biochemistry, and pharmacology. 2018; 47: 293-301.

22. Zhou D, Wu Y, Lin Z, Shi L, Zhao L, Liu T, et al. Prognostic Value of Combination of Pretreatment Red Cell Distribution Width and Neutrophil-to-Lymphocyte Ratio in Patients with Gastric Cancer. 2018; 2018: 8042838.

23. Templeton AJ, McNamara MG, Šeruga B, Vera-Badillo FE, Aneja P, Ocaña A, et al. Prognostic role of neutrophil-to-lymphocyte ratio in solid tumors: a systematic review and meta-analysis. J Natl Cancer Inst. 2014; 106: dju124.

24. Chen L, Hao Y, Cong X, Zou M, Li S, Zhu L, et al. Peripheral Venous Blood Platelet-to-Lymphocyte Ratio (PLR) for Predicting the Survival of Patients With Gastric Cancer Treated With SOX or XELOX Regimen Neoadjuvant Chemotherapy. Technol Cancer Res Treat. 2019; 18: 1533033819829485.

25. Chen L, Yan Y, Zhu L, Cong X, Li S, Song S, et al. Systemic immune-inflammation index as a useful prognostic indicator predicts survival in patients with advanced gastric cancer treated with neoadjuvant chemotherapy. Cancer management and research. 2017; 9: 849-67.

26. Chen L, Hao Y, Zhu L, Li S, Zuo Y, Zhang Y, et al. Monocyte to lymphocyte ratio predicts survival in patients with advanced gastric cancer undergoing neoadjuvant chemotherapy. OncoTargets and therapy. 2017; 10: 4007-16.

27. Zheng HL, Lu J, Xie JW, Wang JB, Lin JX, Chen QY, et al. Exploring the value of new preoperative inflammation prognostic score: white blood cell to hemoglobin for gastric adenocarcinoma patients. BMC Cancer. 2019; 19: 1127.

28. Chen XD, Mao CC, Wu RS, Zhang WT, Lin J, Sun XW, et al. Use of the combination of the preoperative platelet-to-lymphocyte ratio and tumor characteristics to predict peritoneal metastasis in patients with gastric cancer. PloS one. 2017; 12: e0175074.

29. Amin MB, Greene FL, Edge SB, Compton CC, Gershenwald JE, Brookland RK, et al. The Eighth Edition AJCC Cancer Staging Manual: Continuing to build a bridge from a population-based to a more "personalized" approach to cancer staging. CA: a cancer journal for clinicians. 2017; 67: 93-9.

30. Wolchok JD, Hoos A, O'Day S, Weber JS, Hamid O, Lebbe C, et al. Guidelines for the evaluation of immune therapy activity in solid tumors: immune-related response criteria. Clinical cancer research : an official journal of the American Association for Cancer Research. 2009; 15: 7412-20.

31. Wilson D, Hiller L, Geh JI. Review of second-line chemotherapy for advanced gastric adenocarcinoma. Clinical oncology (Royal College of Radiologists (Great Britain)). 2005; 17: 81-90

32. Herrera V, Parsonnet J. Helicobacter pylori and gastric adenocarcinoma. Clinical microbiology and infection: the official publication of the European Society of Clinical Microbiology and Infectious Diseases. 2009; 15: 971-6.

33. Grivennikov SI, Greten FR, Karin M. Immunity, inflammation, and cancer. Cell. 2010; 140: 883-99.

34. Coussens LM, Werb Z. Inflammation and cancer. Nature. 2002; 420:860-7.

35. Balkwill F, Mantovani A. Inflammation and cancer: back to Virchow? Lancet (London, England). 2001; 357: 539-45.

36. Coffelt SB, Kersten K, Doornebal CW, Weiden J, Vrijland K, Hau CS, et al. IL-17-producing gammadelta $\mathrm{T}$ cells and neutrophils conspire to promote breast cancer metastasis. Nature. 2015; 522: 345-8

37. van der Windt DJ, Sud V, Zhang H, Varley PR, Goswami J, Yazdani HO, et al. Neutrophil extracellular traps promote inflammation and development of hepatocellular carcinoma in nonalcoholic steatohepatitis. Hepatology. 2018; 68: $1347-60$.

38. Liu LZ, Zhang $Z$, Zheng BH, Shi $Y$, Duan $M$, Ma LJ, et al. CCL15 recruits suppressive monocytes to facilitate immune escape and disease progression in hepatocellular carcinoma. Hepatology. 2019; 69: 143-59.

39. Veatch JR, Lee SM, Fitzgibbon M, Chow IT, Jesernig B, Schmitt T, et al. Tumor-infiltrating BRAFV600E-specific CD4+ T cells correlated with complete clinical response in melanoma. The Journal of clinical investigation. 2018; 128 : 1563-8.

40. Goode EL, Block MS, Kalli KR, Vierkant RA, Chen W, Fogarty ZC, et al. Dose-Response Association of CD8+ Tumor-Infiltrating Lymphocytes and Survival Time in High-Grade Serous Ovarian Cancer. JAMA oncology. 2017; 3 : e173290.

41. Mei Z, Shi L, Wang B, Yang J, Xiao Z, Du P, et al. Prognostic role of pretreatment blood neutrophil-to-lymphocyte ratio in advanced cancer survivors: A systemic review and meta-analysis of 66 cohort studies. Cancer Treat Rev. 2017; 58: 1-13

42. Pyrhonen S, Kuitunen T, Nyandoto P, Kouri M. Randomised comparison of fluorouracil, epidoxorubicin and methotrexate (FEMTX) plus supportive care 
with supportive care alone in patients with non-resectable gastric cancer. British journal of cancer. 1995; 71: 587-91.

43. Glimelius B, Ekstrom K, Hoffman K, Graf W, Sjoden PO, Haglund U, et al. Randomized comparison between chemotherapy plus best supportive care with best supportive care in advanced gastric cancer. Annals of oncology : official journal of the European Society for Medical Oncology. 1997; 8: 163-8.

44. van Soest RJ, Templeton AI, Vera-Badillo FE, Mercier F, Sonpavde G, Amir E, et al. Neutrophil-to-lymphocyte ratio as a prognostic biomarker for men with metastatic castration-resistant prostate cancer receiving first-line chemotherapy: data from two randomized phase III trials. Annals of oncology: official journal of the European Society for Medical Oncology. 2015; 26: 743-9.

45. Bruix J, Cheng AL, Meinhardt G, Nakajima K, De Sanctis Y, Llovet J. Prognostic factors and predictors of sorafenib benefit in patients with hepatocellular carcinoma: Analysis of two phase III studies. Journal of hepatology. 2017; 67: 999-1008.

46. Choi YH, Lee JW, Lee SH, Choi JH, Kang J, Lee BS,, et al. A High Monocyte-to-Lymphocyte Ratio Predicts Poor Prognosis in Patients with Advanced Gallbladder Cancer Receiving Chemotherapy. Cancer Epidemiol. Biomarkers Prev. 2019; 28: 1045-51.

47. Cao Y, Ni X, Wang Y, Wang L, Yuan K, Gan G, et al. Clinical and prognostic significance of combined plasma fibrinogen concentrations and the monocyte-to-lymphocyte ratio in patients with ovarian cancer. Ann Transl Med 2019; 7: 242.

48. Chi KN, Kheoh T, Ryan CJ, Molina A, Bellmunt J, Vogelzang NJ, et al. A prognostic index model for predicting overall survival in patients with metastatic castration-resistant prostate cancer treated with abiraterone acetate after docetaxel. Annals of oncology : official journal of the European Society for Medical Oncology. 2016; 27: 454-60.

49. Shimada H, Noie T, Ohashi M, Oba K, Takahashi Y. Clinical significance of serum tumor markers for gastric cancer: a systemic review of literature by the Task Force of the Japanese Gastric Cancer Association. Gastric cancer: official journal of the International Gastric Cancer Association and the Japanese Gastric Cancer Association. 2014; 17: 26-33.

50. Chau I, Norman AR, Cunningham D, Waters JS, Oates I, Ross PJ. Multivariate prognostic factor analysis in locally advanced and metastatic esophago-gastric cancer--pooled analysis from three multicenter, randomized, controlled trials using individual patient data. Journal of clinical oncology: official journal of the American Society of Clinical Oncology. 2004; 22: 2395-403.

51. Kim HS, Kim HJ, Kim SY, Kim TY, Lee KW, Baek SK, et al. Second-line chemotherapy versus supportive cancer treatment in advanced gastric cancer: a meta-analysis. Annals of oncology: official journal of the European Society for Medical Oncology. 2013; 24: 2850-4.

52. Sica A, Allavena P, Mantovani A. Cancer related inflammation: the macrophage connection. Cancer letters. 2008; 267: 204-15.

53. Bennett CL, Djulbegovic B, Norris LB, Armitage JO. Colony-stimulating factors for febrile neutropenia during cancer therapy. The New England journal of medicine. 2013; 368: 1131-9.

54. Culakova E, Thota R, Poniewierski MS, Kuderer NM, Wogu AF, Dale DC, et al. Patterns of chemotherapy-associated toxicity and supportive care in US oncology practice: a nationwide prospective cohort study. Cancer medicine. 2014; $3: 434-44$

55. Silber JH, Fridman M, DiPaola RS, Erder MH, Pauly MV, Fox KR. First-cycle blood counts and subsequent neutropenia, dose reduction, or delay in early-stage breast cancer therapy. Journal of clinical oncology: official journal of the American Society of Clinical Oncology. 1998; 16: 2392-400.

56. Cano PM, Vargas A, Lavoie JP. A real-time assay for neutrophil chemotaxis. Biotechniques. 2016; 60: 245-51.

57. Smolen GA, Sordella R, Muir B, Mohapatra G, Barmettler A, Archibald H, et al. Amplification of MET may identify a subset of cancers with extreme sensitivity to the selective tyrosine kinase inhibitor PHA-665752. Proc Natl Acad Sci U S A. 2006; 103: 2316-21.

58. Keir ME, Liang SC, Guleria I, Latchman YE, Qipo A, Albacker LA, et al. Tissue expression of PD-L1 mediates peripheral T cell tolerance. The Journal of experimental medicine. 2006; 203: 883-95.

59. Kang B, Guo RF, Tan XH, Zhao M, Tang ZB, Lu YY. Expression status of ataxia-telangiectasia-mutated gene correlated with prognosis in advanced gastric cancer. Mutation research. 2008; 638: 17-25.

60. Shin EY, Lee BH, Yang JH, Shin KS, Lee GK, Yun HY, et al. Up-regulation and co-expression of fibroblast growth factor receptors in human gastric cancer. Journal of cancer research and clinical oncology. 2000; 126: 519-28. 